\title{
MEASURING THE BALANCE CONTROL SYSTEM - REVIEW
}

\begin{abstract}
Jitka Jančová
Charles University in Prague, Faculty of Physical Education and Sport, Czech Republic: Department of Sport Medicine and Adapted Physical Education

Summary: Past studies of postural control during standing have employed wide range of procedures including the outcome measures use to quantify postural control, the duration of the sample collected, sampling frequency and methods for data processing. Due to these differences there remains little, if any, common grounds for comparisons between studies to establish a concrete understanding of the features and bouns which characterize normal healthy postural control. This article deals with terms such as reliability and repeatability of stabilometric measurements, stabilometric data quantification and analysis. To clear up those terms is suggested, by the author of this paper, very important. The stabilometric measurements remain, nevertheless, different when dealing with aging adults. Though, we notes some alterations of the aging systems, this article is not entirely dedicated to the seniors population. Measurements of COP and technical notes remain the main axis of present paper.
\end{abstract}

Key words: Balance Assessment; Center of Pressure (COP); Center of Mass (COM); Aging; Measurement of Stabilization; Quantification of Balance Control

\section{Introduction}

With the increase in aging population and with increased life expectancy of the elderly, the importance of maintaining mobility, and consequently functional independence, is becomming more and more important. One of factors which determines functional independence, crucial also for quality of senior's life $(26,53)$, is postural stability in the sense of maintaining the upright posture. Moreover, the postural stability is fundamental for every locomotion and manipulation (58). Postural stability (PS) and stabilization is becomming harder with increasing age $(2,40,61)$, when interaction of visual, vestibular and somatosensoric systems is impaired and leads to more frequent destabilization, incertainty and falls $(2,22,39,40,47,61)$. The degeneration of the balance control system in the elderly $(28,65)$, and in main pathologies, has forced researchers and clinitians to understand more about how the system works and how to quantify its status at any point in time. Many studies have shown that postural stability measures correlate with risks of falls, at least in general, even though most falls occur during locomotor tasks and not postural tasks. Notwithstanding, Winter et al. $(38,64)$ add that the ability to stand upright on two feet is important in itself and as a precursor to initiation of other activities of daily living, especially for seniors. Unfortunately injuries and loss of life due to falls in the elderly are a very major factors facing them. Fear of falls is than a common cause of decreased physical activity accompanied by decreased muscle strength of lower limbs, which causes in return falls again $(5,12,41,61)$. Next consequences are described as loss of functional independence $(54,56)$, loss of quality of life $(5,23,56)$ and death $(43)$. Society's concern should be focused on the elderly and their extreme death rate due to falls. If more than one-third of adults over 65 fall each year (20) and these falls are seen as the primary cause of death and injury among this population, we see the necessity of monitoring of PS in the elderly. Objective measurements were proposed by stabilometric methods (SM) with standardized tests of standing $(9,24$, $28,29,49,53,57,61)$. The purpose of this review is to report about the stabilization and its measurements that is seen in the related literature destinated to balance control and it's quantification.

\section{Theoretical Review}

Major notions used in this review are terms such as Posture, Balance and Stabilization, Body Sway, Center of Pressure (COP), Center of Mass (COM), Ankle Strategy, Inverted Pendulum etc. That is why we will see them more closer one by one. Further in this chapter we deal with the somatosensory systems that contribute to our stabilization during both: quiet standing and locomotion.

Posture describes the orientation of any body segment relative to the gravitional vector. It is defined also as an angular measure from the vertical (64). Nevertheless, we prefer 
to use the term posture as a characteristics of human body maintained (balanced) in upright standing position (58).

Balance may be seen as a generic term describing the dynamics of body posture to prevent failing (64). It is related to the inertial forces acting on the body and the inertial characteristics of body segments and to the tightness of neural control loop involving receptors and effectors and uncontrolled neural activity (noise) under the condition of unstable dynamic state (see inverted pendulum). The result of balance is stabilization. Stabilization as a term involves process and its inter-individual variability. Good ability of stabilization may be also perceived as reduced body sway. Stabilization is an important departure point for every locomotion and manipulation (58).

Body sway is a kinematic term and is often estimated from Center of Pressure (COP) measures derived from force plate data, and even eroneously assumed $(60,65)$ to be synonymous to COP measure.

Center od Pressure (COP) is the center of the distribution of the total force applied to the supporting surface. It is a point location onto the vertical ground reaction force vector. It represents a weighted average value of all pressures over the surface of the area in contact with the ground. If one foot is on the ground the COP lies within that foot, if both feet are on the the ground COP lies somewhere between the two feet, depending on the relative weight taken by each foot. The location of the COP under each foot is a direct reflection of the neural control of the ankle muscles (64). Increasing plantaflexors activity moves the COP anteriorly, increasing invertor activity moves it laterally. Its units are meters (64).

Center of Mass (COM) is a point equivalent (64) of the total body mass in the global reference system (GRS) and is the weighted average (64) from COM of each body segment in $3 \mathrm{D}$ space. It is a passive variable controlled by the balance control system. The verical projection of the COM onto the ground is often called the Center of Gravity (COG). Its units are in metres. By ones the COM is reported (30) to be totally independent of the COP, others consider that $\mathrm{COM}$ in quiet standing is projected to the COP.

Ankle strategy so called by Winter (64) "epicenter" of radiation of responses when body balancing while quiet stance and during small perturbations. Predicts that the ankle plantarflexors/dorsiflexors alone act to control the Inverted Pendulum $(6,46,64)$. Thus it appears that the CNS recognizes the needs to stabilize the joint closest to perturbation first, followed by the knee, hip, and spine. Because these responses radiated from the ankle towards the body's COM, those responses were described as an ankle strategy. An alternate strategy, called a hip strategy was identified when the ankle muscles were unable to respond (because the foot was positioned sideways i. e. on a narow beam) (64). In more perturbed situations or when the ankle muscles cannot act a hip strategy would respond to flex the hip, thus moving the COM posteriorly, to extend the hip to move the COM anteriorly $(6,46,64)$.
The A/P balance is usually dominated by hip controll 0.71 with mixed, small and sometimes negligeable contributions by the ankle plantar/dorsiflexors 0.22 (64). Ankle inventors/evertors dominate 0.99 with mixed and small contribution from the hip load/unload mechanism 0.31 (64). The A/P control of the balance requires collaboration between right and left plantarflexors and dorsiflexors. All the invertors are also plantarfelxors and dorsiflexors. We recognize:

- Ankle invertors (mm. tibialis anterior and posterior, extensor digitorum longus, and hallucis longus).

- Ankle evertors (mm. peroneii).

Both (evertors and invertors) cannot act independently. The COP under each foot will move back and forth in almost complete synchronisation, however any $\mathrm{M} / \mathrm{L}$ component by the invertors/evertors will move the COP under both feet in the same medial or lateral direction. Prince, Winter, Archer (46) realized the motor responses in the M/L direction were totally dominated by a hip load/unload strategy. This hip strategy was completely independent of the ankle strategy which as expected totally dominated the $\mathrm{A} / \mathrm{P}$ balance control. The CNS is of highest importance, because it is controlling a multisegmental system and the interlimb coupling that play an important role for balance control.

Inverted pendulum is, in the frontal line, a parallelogram pivoting about both ankle and both hip joints, and is under the control of four sets of muscles. In the sagital plane the pendulum pivots about the ankle in quiet stance or about both hips and ankle in perturbed standing. The inverted pendulum (as a biomechanical model of balance) can be used to explore how the CNS controls balance. Especially in the kinetics of human movement we may see there the integrated control evident at each joint and in entire limb. $(6,46,60,62,64,65,68)$.

Three major sensory systems are involved in balance and posture:

Vision - the visual system is a major contributor to balance, providing information about the environment and the location, direction, and speed of movement of the individual (53). Because many postural reflexes triggered by the vestibular system can also be triggered by stimulation, vision and compensate for some loss of vestibular function. However, in most very old individuals, vision is also degraded and provides decreased or distored information. Consequently the poor visual acuity is correlated with the number of falls older people experience (55). With aging people often lose the ability to detect spatioal information that would assist in balance. They also progressively loose their peripheral vision. Peripheral vision makes a very important contribution to the control of anterior posterior sway of the body (66) and its absence leads to a greater number of falls. Benson (2), Winter, Prince et al. $(60,65)$ stated there was essentially no difference between the eyes open and eyes closed conditions during quiet standing. Another approache developed by Chiari et al. $(8,10)$ showed 
that vision had a clear effect on the correlation between parameters and biomechanical factors. This correlation was typically higher with eyes closed than with eyes open and could be interpreted as a major influence of body biomechanics on postural sway upon eyes closure. The loss of visual input has been shown to force, $\mathrm{n}$ most subjects, an increase muscle stiffness.

Vestibular system - Located in the inner ear, is a system of receptors that provides information about movements of the head. Otoliths provide a static vertical reference during postural standing and signal the head's position with respect to gravity. Another type of sensors are the semicircular canals aligned with the three planes of the body: frontal, sagital and horizontal. These canals are filled with a fluid that moves in response to head movements. The receptors that are triggered by this fluid movement than provide information about the turning of the head. The neurons of both of these vestibular structures have powerful direct influences over the motor neurons in the spinal cord that activate muscles(especially extensor muscles) and thus contribute substantially to balance. Vestibular neurons decrease both in numbers and in size of nerve fiber wih aging, beginning at about age 40 . Persons over age 70 may have lost $40 \%$ of the sensory cells within the vestibular system. With more advanced aging, the sensitivity of the peripheral receptors of the vestibular system is reduced $(26,53,56)$.

Somatosensory system - this system is critical to balance and motor control, it provides information related to body contact and position. It includes cutaneous receptors that provide information about touch and vibration and muscle receptors that provide information about the position of the limbs and body. Muscle receptors also signal changes in limb and body positions. The control of movement is dependent upon constant and accurate information from the somatosensory system $(26,53,56)$.

Cutaneous receptors signal when any mechanical stimulus is applied to the body surface. Thus when the skin is contacted and changes in pressure on the skin occur, neural impulses are directed centrally. The importance of this information is fully appreciated when one experiences the difficulty of balancing or walking when this information is absent $(26,53,56)$. Normal individuals often experience the loss of these receptors when they sit in one position for a long time, restricting the blood supply to the lower limbs. Clearly the contact of the skin with the shoes and the changes in pressure result as the body weight shifts heel to toe are important sources of information in the maintenance of balance. Cutaneous sensations grows less sensitive with aging. Although the ability to detect cutaneous and vibratory stimuli is greatly influenced by disease and nutrition, it also declines significantly with aging (51). The major contribution, represented for the elderly in fear of falling, leads us to research in the field of postural stability and its alteration. The most important question, in this moment, is subsequently how the postural stability may be controlled.

\section{Control of the postural stability}

From the dynamic point of wiew, stability may be defined as a quantitative response of a system's state variables (i. e. position, angles, velocities, etc.) to perturbations (16).

Wooly et al. (65) demonstrates that balance control, even during quiet standing, is not a simple single motor control pattern but a synchronised effort between two independent motor groups. The role of these balance mechanisms is important in the study of falls especially in the elderly. The result of the neromuscular control is COP which reflects the combined control of both left and right dorsiflexors/plantarflexors (52) by Spaepen called COP ${ }_{\text {net }}$. We do not know whether the left and right foot control is symetrical and in unilateral pathologies and even in normals there is a dominant limb control (when using two force platforms we may see this independent left and right control at the ankles, and may also see a totally separate control of balance in $\mathrm{M} / \mathrm{L}$ direction). It might be reasonable, therefore, to assume that the mechanism used to maintain the stability of standing and walking should be quite different. Standing and walking are reported to be wery different tasks (27).

Generally, those results offer converging evidence for the existence of self-organised phenomena operating at the level of whole body coordination and encourage further examination of the possibility that the interactions between the various components of the postural system might be addressed through the physics of nonequilibrium processes, see Megrot et al. (40).

Quiet standing has been the subject of many research papers. Balance control is chalenging task for biped humans since the large mass (2/3 of body mass) of the head, arms, and trunk (HAT) is located a fair distance $(2 / 3$ of body height) over relatively small base of support (BOS). Maintaining an unstable equilibrium requires that multiple joints must be coordinated so that the COM is kept above the base of support (40). Locomotion studies have provided some insight in the undrestanding of the human locomotor control systems (37). Activation of this system ensure i. e. the initiation of gait. The postural balance control of the upper body in the frontal and sagital planes in steady state gait has been reported by MacKinnon and Winter (39). Winter, Prince et al. (46) state that balance control even during quiet standing is not a simple single motor control pattern but is a collaborative effort between two independent motor groups. The role of these balance mechanisms is especially important in the study of falls in the elderly. A loss of balance in the lateral directions is very difficult to recover because the unloaded limb is on the opposite side from the directions of fall, whereas in the $\mathrm{A} / \mathrm{P}$ direction a corrective step forward or backward is possible.

Megrot (40) made a part of his measurements on an unstable platform and his participants had to maintain their balance while he tried to see how the underlying dynamics of local (foot, hip or head) and global centre COM compo- 
nents are involved in balance control and are affected by changes in the available information. However, in the $\mathrm{M} / \mathrm{L}$ direction the COP is controlled by the hip abductors/adductors via the load/unload mechanism and these changes varies inversely with stance width as reported by Winter et al (62).

Author of this paper actually speculate over the question if an intervention focused on local breathing and using diaphragm, increasing the intra-ventral pressure, is going to help by distributing the balance uniformly on both feet. Recording from each limb separately allows to us to identify a separate load/unload mechanism for controlling the $\mathrm{M} / \mathrm{L}$ COP (68) while A/P balance is controlled independently by the plantar dorsiflexors. For all measurements is very important to standardize the tests of quiet standing. Which are many and for this reason we will see more closely only some of them, whose importance has been revealed by many authors.

\section{Tests of quiet standing}

- Tandem stance: (right or left foot is leading)

- Side by side stance (parallel narow, or parallel broad)

- 45degr. stance (right foot is ahead of the left foot and at a confortable distance to the side).

- Romberg stance (keeping balance with eyes closed, feet in paralel position)

On each platform and with each type of stance, subjects are instructed to place their arms naturally at their side and to maintain the position for measured time with eyes open or closed. Yunacheng et al. (68) reported that during quiet stance with feet side by side on two different platforms, the motor responses in the $\mathrm{M} / \mathrm{L}$ direction were totaly dominated by a hip load/unload strategy. This hip strategy was completely independent of the ankle strategy which, as expected, dominated the A/P control. However after this recent partitioning of an independent ankle (labeled $\mathrm{COP}_{\mathrm{c}}$ ) and hip (labeled $\mathrm{COP}_{\mathrm{v}}$ ) mechanism in the $\mathrm{A} / \mathrm{P}$ and $\mathrm{M} / \mathrm{L}$ direction respectively, the authors are prompted to examine these mechanism in other foot configuration. COP profiles demonstrated an opposite trend to those found in the sideby-side stance configuration than to tandem stance configuration. Winter et al. (33) determined also the relative role of each of neural control strategies when the subject adopted different foot position: tandem stance, and an intermediate position called $45 \mathrm{degr}$., half way between the tandem and side by side position. Romberg type of stance is not recomended for children before the age of 4 (for the reason, that they are not able to make it), and more difficult postures with occluded vision before the age of 6 . According to the litterature the adequate performance of this task is contingent upon the myelinisation of the ponto-cerebellar pathways, which are an essential part of the neo-cerebellocortical connection circuits (11). Winter, Prince et al. (33, $38,46,60,65)$ state that on the stance position taken, depend many combinations of an ankle mechanism and a hip (load/unload) mechanism which are evident. In side by side stance, the A/P balance is totally under ankle (plantar/dorsiflexor) control, whereas $\mathrm{M} / \mathrm{L}$ balance is under hip (abductor/adductor) control. In tandem stance, the A/P balance is dominated by hip mechanism, with mixed or sometimes negligible contributions by the ankle plantar/dorsiflexors: for $\mathrm{M} / \mathrm{L}$ balance the reverse is evident. Ankle invertors/evertors dominate, with mixed and small contributions from the hip load/unload mechanism. In an intermediate 45 degr. stance position, both ankle and hip mechanisms contribute to the net balance control in totally different ways. Nevertheless, quantifying posture requires modelling the body with a sufficient number of body segments and precise determination of the position and orientation of each body segment. During quiet standing the variables of both shall not differ. The most common position of the feet is a side-by-side position and the most commonly discussed control is in the $\mathrm{A} / \mathrm{P}$ direction using an "ankle strategy". With the standardization of tests of quiet standing we may consider important to continue with the standardization of COM, COG and COP and its records.

\section{COP versus COG}

The difference between the COP and COG has been recognised by many researchers $(1,3,7,46,52,60,65)$. Researches are often estimating the location of the total body COM and some authosr are tracking its trajectory over the course of time (52). Riley, Mann, Hodges (40) add that the whole body COG is estimated and compared to concurent force plate Center of Force $(\mathrm{COF})$ data $(\mathrm{COF}=\mathrm{COP})$. $\mathrm{COG}$ and $\mathrm{COP}$ excursions agree where dynamics are not significant. The technique may be employed to study quiet stance, response to postural disturbances, or the initiation and coordination complex movements such as gait. The posture, position and orientation of the body segments, and balance, the control of the COG or COF (=COP), are coupled. Postural adjustements change the most the COG location. These models are useful for describing quasi static stance and responses to small disturbances of stance posture and balance. They are, however, too restrictive to represent the range of postural control strategies employed in complex activities such as walking, rising from a chair or the precise coordination.

\section{COP versus COM}

The interaction between the COM and COP is tightly regulated to control total body balance. Coarse control of this balance is achieved by foot placement, with fine control during weight bearing by the ankle musculature $(18,39$, 68).

Yunacheng et al. (68) state that if the body moves as an inverted pendulum, a linear relationship between the $\mathrm{COP} / \mathrm{COM}$ vector should be present while standing, and the horizontal acceleration of COM, while walking. MacKin- 
non and Winter (39), report also that the patterns of the COM versus the COP trajectory are very important in body balance control especially during transient gait. By the initiate phase of gait was interested also Breniere and Do (4). Their interest was focused mainly to the question when and how the stationary process of gait begins and when walking starts from upright posture.

While standing movements of the COP approximate those of the COG only during postures that are nearly static or quasi static (19a).

Winter et al. (23) hypothesised that traditional COP measures of standing balance would be correlated with local dynamical stability of standing but not of walking. The lack of any such correlations between COP measures and walking parameters further supports the idea that the mechanism governing the local dynamic stability during standing and walking are inherently different.

Winter (64) proved for all trials the correlation between the (COP-COM) signal at the horizontal $\mathrm{A} / \mathrm{P}$ and $\mathrm{M} / \mathrm{L}$ acceleration averaged -0.79 . However in the $M / L$ direction COP was significantly less that in the A/P direction and decreases significantly as stance width increases.

\section{COP records}

Clinical and experimental assessments of balance use measures of COP excursion to quantify postural stability during standing. Hasan and Robin (19 a, b) asume that the greater COP excursions, the greater the imbalance. The primary biomechanical variable that has been recorded to quantify the time course of postural balance during quiet standing, has been the COP removed from noise and called $\mathrm{COP}_{\text {net }}$ expressed in the $\mathrm{A} / \mathrm{P}$ and $\mathrm{M} / \mathrm{L}$ directions. Winter et al. (62) calculated the COP net in $\mathrm{A} / \mathrm{P}$ and $\mathrm{M} / \mathrm{L}$ directions as follows: COP - due to ankle mechanism, and COP due to hip load/unload mechanism. By using the single force platform, the researchers were not able to discriminate the participation of each individual foot in maintaining balance (46) in contrary to records measured on two platforms. Hassan and Robin (18) presented a study, where they tempted to describe the normative characteristics and stance dependent differences between the anterioposterior and mediolateral componets of both. Winter et al. $(46,60,65)$ report on the COP displacement during upright standing abound, also says: "that the data simultaneously measured on COP and COG can be compared". Little et al. (38) predict an initial mechanical response of the COP to be in phase with the angular changes, which would than be augmented by the reflex response after the neuromuscular delays, which has been experimentally confirmed. However, researchers report that many measures can be derived from the COP data $(32,34)$. Breniere and Do (4) report that it was easy to calculate the instantaneous coordinates of the COP, which was defined by the authors as the barycenter of the vertical ground forces measured by the vertical transducers. The precision of force determination was half Newton, and for displacement of COP, was less than $3 \mathrm{~mm}$. With COP records we must stress the quantification of the data recorded.

\section{Quantification of the data}

Winter, Prince et al. $(33,38,46,60,65)$ quantify the control of the posture in quiet stance by COP changes in the $\mathrm{A} / \mathrm{P}$ and $\mathrm{M} / \mathrm{L}$ directions from a single force platform. The same authors (60) use a spatial plot of the separate mechanism which reveals the fact that the random-looking COP scatter plot is nothing more than a spatial plots. Koozenkanani and Duerk (61) tried to compute COP excursions from kinematic records obtained during voluntary sway. They showed that the COP excursions computed using the model were much more comparable to platformderived COP than those computed using anthropometric databases compiled from cadaver studies.

Kang and Dingwell quantified the local dynamic stability from the mean divergence over time of locally perturbed trajectories in state space, which were parameterized as a double exponential process (27). Divergence parameters were compared to determine the relationship between local dynamic stability during standing and walking. Divergence parameters were also compared to traditional COP measures obtained from standing trials.

Using dimensional analyses (largest Lyapunov exponent and correlation dimension), Megrot et al. (40) examined the active degrees of freedom involved in balance control. Results indicated a similarity in dimension between a fixed point and a limit cycle. The behavior of COM was found to be more predictable than the behavior of its local constituents.

Winter et al. (62) remind that root mean squares (RMS) COP measures in $\mathrm{A} / \mathrm{P}$ direction were the same for all three stance widths, which was not surprising because the A/P base of support does not change with stance width. Also cross-correlations were used by Winter et al. (46) to compare between the foot configuration. The RMS of COP ankle and COP hip over the two minutes stance were also calculated. The MPF of the COP and (COP-COM) were calculated in both $\mathrm{A} / \mathrm{P}$ and $\mathrm{M} / \mathrm{L}$ directions.

Other authors such as LeClair, Riach (35) found RMS significantly influenced by sample duration in both the $\mathrm{A} / \mathrm{P}$ and $\mathrm{M} / \mathrm{L}$ directions. Mean power frequency (MPF) was also influenced by sample duration in both $\mathrm{A} / \mathrm{P}$ and M/L directions. Unlike MPF and RMS, calculted values of MPOS (Mean Position) (35) were not significantly influenced by sample duration in either the $\mathrm{A} / \mathrm{P}$ or $\mathrm{M} / \mathrm{L}$ directions. According to Freedman (15) a transient component of the COP signal is contained primarilly in the first 20 s of the time series. Based on this information, the initial transient component of a 10 s sample may be different from a 20 s sample measured from the same subject. With those components we enter the chapter of data analysis and the importance of sampling frequency. 


\section{Data analysis}

Winter, Prince et al. proposed $(60,65)$ a relatively simple control scheme for regulation of upright posture that provides almost instantaneous corrective response and reduces the operating demands on the CNS. They derived and experimentally validated an analytic stiffness model which was developed in quiet standing. The model assumes that muscles act as springs to cause the COP to move in phase with the COM as the body sways about some desired position. In the sagital plane this stiffness control exists at the ankle plantaflexors in the frontal plane, by the hip abductors/adductors.

LeClair and Riach (35) collected the ground reaction forces and moments in three planes at a sampling frequency of $20 \mathrm{~Hz}$ and converted to a digital signal via a 16-bit $\mathrm{A} / \mathrm{D}$ converter. Continuous displacement of $\mathrm{COP}$ was analysed as eight consecutive $15 \mathrm{sec}$ records, four consecutive $30 \mathrm{sec}$ records, two consecutive $60 \mathrm{sec}$ records and the entire $120 \mathrm{sec}$ record of COP displacement. For each of the respective samples, the signal was filtered at $5 \mathrm{~Hz}$ using a dual pass Butterworth filter and measures of RMS, MPF and MPOS were calculated in both $\mathrm{A} / \mathrm{P}$ and $\mathrm{M} / \mathrm{L}$ directions. RMS were derived following removal of the bias value from each signal, in this case RMS is equivalent to the standart deviation of the COP displacements. The same authors also confirmed the significance of MPF values for longer samples, which represents the contribution of the low frequency component that cannot be detected within the shorter samples. In addition to improving the ability of to detect the contribution of the low frequency components within the COP signal, the use of longer duration samples also increases the resolution of the FFT (Fast Fourier Transformation). Measures, differed in time recording, taken by LeClair and Riach (35) measures were collapsed across trials and were entered into a one way, within subject repeated measures analysis of variance ANOVA to test the effects of sample duration. In the case of significant main effects of sample duration, Bonferroni t-test were used to determine significant differences between pairs of sample duration with a level of significance of 0,05 .

ANOVA procedure and linear correlation was incorporated, correlation coefficients ( $r$ ) were computed to test the degree of coherence between different parameters in order to consider the inter-individual variation, a statistical significant level at $p \leq 0.01$ was chosen, associated to a FisherSnedecort criteria (4).

LeClair, Riach (35) analysed as well significant differencies, by means of one way within subject repeated measures ANOVA, where any variability due to dependence between levels of time was accounted for. Lenhoff et al. (36) reported on traditional statistical method used to analyse gait data and calculate either prediction or confidence intervals at a set of time points across the gait cycle. A computationally intensive statistical method which calculates simultaneous intervals (called bootstrap) for gait data has a significant advantages over the traditional methods. In addition to producing simultaneous intervals, the method they advocated was non-parametric in that it does not suppose a parametric form for the underlying curves being analysed.

\section{Sampling frequency and it's importance}

According to Hasan et al. (19b) MPF is a summary measure of the power spectrum, frequently used in electromyographic studies of muscle fatigue, and is given as:

$$
\mathrm{MPF}={ }_{\mathrm{fn}} \sum_{\mathrm{f}=0} \mathrm{fP}(\mathrm{f}) /{ }^{\mathrm{fn}} \Sigma_{\mathrm{f}=0} \mathrm{fP}(\mathrm{f})
$$

Past studies of postural control during standing have employed wide range of procedures including the outcome measures use to quantify postural control, the duration of the sample collected, sampling frequency and methods for data processing (17). Even though, a little research has been dedicated to examine how factors, such as sample duration or a sample frequency may impact COP summary measures in both time and frequency domains, despite evidence for time varying characteristics of the COP signal. However, only a limited number of studies has been performed to directly examine the effect of sample duration of COP outcome measures (37).

Furthemore, the collection of long duration COP records (more than $60 \mathrm{sec}$ ) has revealed that high frequency adjustements of COP responsible for controlling movements of the COM are superimposed upon very low frequency oscillations that are not detectable when sampled for shorter periods of time $(13,44,63)$. For that reason also FFT becomes hard to use. Full et al (16) and Prieto et al. (45) presented the excursion peak-to-peak ranges, RMS, and MPF in $\mathrm{A} / \mathrm{P}$ and $\mathrm{M} / \mathrm{L}$ directions $95 \%$ confidence interval elipse area covering the COP trajectory, and mean sway velocity were calculated. Winter et al. (62) realised that in order to determine the harmonic content of COP and COM, also the length of the record needs to be taken in consideration. When we finally look at the error signal (COP-COM) we see a dramatic increase in the MPF. This is because the tCOP "tracks" the low frequency COM fluctuation and when the COM is substracted from the COP. These very low frequencies are largely removed leaving the higher frequency control signal. There were no significant differences in the MPF's in the A/P direction. However, in the M/L direction the MPF at $150 \%$ was $0.917 \mathrm{~Hz}$ which was significantly higher than the $0.634 \mathrm{~Hz}$ in the $50 \%$ position. Nevertheless, Winter et al. (62) add that the MPF reported for COM cannot be compared with their previous work because only COP were measured: "our MPF of the COP are dramatically lower than other reports because the duration of our trials $(2 \mathrm{~min})$ permitted analyses of the large amplitude low frequencies not possible in previous work (30 sec)". Carpenter, Winter et al.(6) present a study which purpose was to examine the effect of sample duration on 
the magnitude and reliability of COP summary measures commonly used to characterise the control of upright stance. Each record was subdivided into 15, 30, 60, $120 \mathrm{sec}$ samples and RMS, MPF and mean position of COP were calculated for each sample. RMS significantly increased and MPF values significantly decreased as sample duration increased. Substantial increases in the reliability of both RMS and MPF were observed with increased sample duration. LeClair, Riach (35) attributed the sensitivity of RMS and MPF of increasing sample duration, at least in part, to the representation of low versus high frequency components of the power spectrum (62). For the same author it appears that the bias or MPOS value of COP during quiet stance remains relatively constant over time. Perhaps such characteristics are not surprising if the goal of the central nervous system is viewed as maintaining the body COM and the controlling COP at some constant position (bias) within the base of support (BOS). But not only the quantification and statistical analysis remain important for measurements on stabilometric platforms. The other, very strong component, is represented by the repeatability and reliability of those measurements. Those variables will be discussed in next chapter.

\section{Repeatability of the kinematic data and the reliability of foot pressure measurement}

Repeatability of the kinematic data is an uneasy problem. Everybody has its individual stance which depends on many variables. Kidder et al. (31) were aware that it was essential to determine the repeatability of balance and gait analysis system when evaluating its effectiveness in clinical observation and decision making. In 1989 Kadaba et al. (25) investigated the repeatability of kinematic, kinetic and elektromyographic measurements made with a clinical lower body gait analysis system. A coefficient of multiple correlation (CMC) was used to express the repeatability of the system for within and between day testing. As result it should be noted that within day repeatabilities compare well with values for lower body gait analysis, and the foot and ankle system demonstrates better between day repeatability. Bong et al. (3) evaluated the repeatability by means of the coefficient of variation for time and distance parameters at each important event in the gait cycle in sagital, corronal and transversal plane.

Repeatability of the kinematic data in the quantitative motion analysis were acceptable in 10 repetitions of trial in 10 healthy subjects and were remarkably best in sagital plane folowed by the coronal and transversal planes in decreasing order. LeClair and Riach (35) attempted to address the question of optimal sampling duration for COP summary measures of time domain with intention to see, how repeatable is such a measure across successive trials when generated from a particular sample period. Independently on software products or optimal sampling duration, the repeatability depends much more on psychological and emotional state of each and every individual. We use to take in consideration the fact that posturography measurements alow us to analyse only the instantaneous posture.

According as we measure the repeatability we may conclude on reliability. Generally, we may state that the values of the correlation are not too high (24) Although the ways and patterns which characterize human beeings in their endeavour to maintain body equilibrium can be easily observed and described. The objective meaurement of postural control, meets the standards of scientific reliability and validity, and are a matter of considerable sophistication involving series of methodological problems (60).

We also often deal with the question whether the reliability is better within the same day or between (i. e.) two days. On one hand Castagno et al. (7) reports that the reliability of repeated foot pressure measurements within the same day has not been established. On the other hand LeClair and Riach (35) came out with an intraclass outcome correlation coefficient, used as a measure of reliability and calculated from three consecutive trials for each of the dependent measures from the initial 15, 30, 60 and 120 sec samples. Foot pressure measurements were obtained for each subject in three trials on five separate days. His results indicated that the heel, forefoot and total areas of the foot produced reliability coefficients greater than 0.7 for within visit and between visit. Nevertheless, we haven't seen any confirmation from other authors if repeating the same measurement. Samson and colleagues (50) studied the intrasession reliability of various biomechanical variables of postural control in a quasi static position. The study measured the length of the COP trajectory in healthy people between 20 and 60 years of age. It used a questionable index for estimating reliability $\mathrm{SD} /$ mean $\mathrm{x} 100$ and proposed to average 10 repetitions to improve the reliability. However this number of repetitions was not justified.

Therefore, it is crucial to know the signal-to-noise ratio; the noise causes the unreliability of the variable measured (COP). The measurement error should be taken into consideration when setting the clinically important difference. Not only Hebert and colleagues (21) assume that this difference should be greater than the measurement error. They comment that the reliability is influenced differently by sample durations for different summary measures. Their observation would suggest that a sample duration (at least $60 \mathrm{sec}$ ) should be used to optimize the stability and reliability of summary measures of COP during quiet stance. Using longer duration records increases, on one side, the reliability of COP, and therefore may increse the possibility of discovering more subtle differences between groups of postural strategies. But on the other side COP records of short duration capture only the high frequency components of the signal which reflects COP control over Center of Mass (COM). There is important to suggest that in quiet stance the COP reflects the COM. According some authors the COP can be compared to COM (61) and according to other authors even equated (62). This may be important to 
consider when trying to use the COP summary measures to characterize some pathologies and control strategies. From the literature review it is obvious that the estimation of reliability has fundamental impact on whole posturographic research. As we reported already in Acta Medica 2007 (24) Fleiss (14) offered opinion about acceptable reliability for tests, proposing guide as folows:

- over 0.79 as high

- 0.40-0.79 moderate

- less - poor

But the other authors suggested a more rigorous acceptable coefficient of reliability for tests, i.e. Kelley (30) recommanded a minimum of 0.94, Weiner and Stewart (59) suggested 0.85 , unfortunatelly they did not indicate how their reliability was measured, so we were not able to make an equivalent measurement, nor the approximation of reliability, reported as well in Acta Medica in 2007 (24). We may state that the range of body sway may increase with every trial i. e. due to fatigue. Those who are less stable in $\mathrm{A} / \mathrm{P}$ direction in the first test, show even larger range in the other tests. Those who have the tendency to stand on the front part of the feet, show the same tendency in the other tests. The best values, in the sens of stability and reliability, were present during the first test. Fatigue is one of the possible explanations. Trials when subjects are standing with eyes closed show nearly the same body sway as trials with eyes open. We may also consider it as a factor related with youth. We, however, are not about to make a definite statement, before repeating the same trials with elderly, as did Chiari et al. (8) for whom the vision had a clear effect on the correlation between body sway and higher age. Chiari's correlation was stated as typically higher with EC than with EO, and can be interpreted as a major influence of body biomechanics on postural sway upon eyes closure. In this condition the inertial properties of the body, dependent on height and weight, may become preponderant because of the removal of the visual afferent input to the postural control system. In fact, the loss of visual input has been shown to force, in most subjects, an increase of muscle stiffness (8) which insreases body sway respectively.

\section{Conclusion}

From our point of view the above presentation of posturographic measurement results is rather controversial. The general trend is to prefer higher values of reliability coefficient, even when its estimation is kept unpublished. Therefore, it is difficult to say the given reliability coefficients are not only real but really repeatable (24). The importance to assess the repeatability and reliability of stabilometric measurements is evident especially for the reason that if measures of COP are to be used for the diagnosis of pathological population and /or monitoring rehabilitation progress, then the summary measures that are used to quantify changes in COP must not only be stable but also reliable. It is, notwithstanding, clear that stabilometry with its advantages and also its inconvenients, remain an important approach to standardization of postural stabiliy with respect to its other, not less important components. As conclusion for our needs results that stabilometry is considered as an integral part of procedures when assessing the PS, especially efficient when dealing with the elderly. For our further research results also, that we may subsequently work separately with parameters (indicators) of PS in order to say whether aging influence more the utmost values of $\mathrm{M} / \mathrm{L}$ or A/P sway or the velocity of titubation. The second is considered by the author of this paper as most characterized by regression changes in the aging organism, which is to be confirmed.

\section{References}

1. Benda BJ, Riley PO, Krebs DE. Biomechanical relationship between center of gravity and center of pressure during standing. IEEE Trans. on Neural Systems and Rehabilitation 1994; 2(1):3-10.

2. Benson AJ, Spencer MB, Stott JR. Tresholds for detection of the direction of whole body linear movement in the horizontal plane. Aviat Space Environ Med 1986;57: 1088-1096.

3. Bong QK, Kim MD et al. Reliability of kinematic data in normal adult gait. Gait and Posture 1996;4:196

4. Breniere Y, Do MC. When and how does the steady state gait movement induced from upright posture begin? Journal of Biomechanics 1986;19:1035-1040.

5. Brouwer B, Musselman K, Culham E. Physical function and health status among seniors with and without fear of falling. Gerontology 2004;50:135-14.

6. Carpenter MG, Frank JS, Winter DA, Peysar GW. Sampling duration effects on COP sumary measures. Gait and Posture 2001;13:35-40.

7. Castagno P, Miller F, Richards J, Gaboury L, Lennon N. Reliability of foot pressure measurements in clinical gait analysis. Gait and Posture 1996;4:170.

8. Chiari L, Bertani A, Capello A. Classification of visual strategies in human postural control by stochastic parameters. Human Movement and Science 2000; 19:814-842.

9. Chiari L, Capello A, Lenzi D, Della Croce U. An improved technique for the extraction of stochastic parameters from stabilograms. Gait and Posture 2000; $12: 225-234$.

10. Chiari L, Rocchi L, Cappello A. Stabilometric parameters are affected by anthropometry and foot placement. Clinical Biomechanic 2002;666-677.

11. Cohen Raz R. Learning disabilities and postural control, Freund Publishing House LTD, London 1996.

12. Cumming RG, Saltkeld G, Thomas M, et al. Prospective study of the impact of fear of falling on activities of daily living, SF-36 Scores, and nursing and home admission. Journal of Gerontology in Medical Science 2000; 55A: M229-M305.

13. Duarte M, Zatsiorsky VM. Patterns of COP migration during prolonged unconstrained standing. Motor control 1999;3:12-27.

14. Fleiss JL. The design and analysis of clinical experiments. New York: John Wiley and Sons, 1986

15. Freedman C. Non stational properties on postural sway. Journal of Biomechanics 1993;26:409-416.

16. Full RJ, Kubow T, Schmitt J, Holmes P, Koditschek DE. Quantifying dynamic stability and manoeuverability in legged locomotion. Integrative and Comparative Biology 2002;42:149-157.

17. Harris GF, Riedel SA, Matesi D, Smith E. Standing postural stability assessment and signal stationarity in children with cerebral palsy. IEEE trans Rehabil Eng 1993; $1: 35-42$.

18. Hasan SS, Robin DV. Simultaneous measurement of body COP and COG during upright stance. Part II: Amplitude and frequency data. Gait and Posture 1996;11-20.

19. a. Hasan SS, Robin DV et al. Simultaneous measurement of body COP and COG during upright stance. Part I: Methods. Gait and Posture 1996;11-20. b. Hasan SS, Robin DV et al. Simultaneous measurement of body COP and COG during upright stance. Part II: Amplitude snd frequency data. Gait and Posture $1996 ; 11-20$.

20. Hausdorff JM, Ashkenazy Y, Peng CK et al. When human walking becomes a random walking: fractal analysis and modelling of gait rhythm fluctuations. Physica A: Statistical mechanics and its applications 2001;302:137-147).

21. Hebert R, Springerhalter DJ, Brayne C. Setting the minimal metrically detectable change on disability rating scales. Archives of physical medicine and rehabilitation 1997;78:1305-1308.

22. Horak FB, Diener HC. Cerebellar control in postural scaling and central set in stance. Journal of Neurophysiology 1994;2:479-49. 
23. Howland J, Lachman ME, Peterson EV et al. Covariates of fear of falling and associated activity curtailment. Gerontology 1998;38:549-555.

24. Jančová J, Tošnerová $V$. Use of stabilometric platform and evaluation for futher measurements - a pilot study. Acta Medica Universitatis Carolinae 2007;50(2):139-143

25. Kadaba et al. Journal of Orthopaedic Research 1989;849-860 In: Kidder SMS, Abuzzahab FMS, Dow A Ortiz TBS, Harris G, Johnson JMD Repeatability of postural data in normal foot and ankle motion. Gait and Posture 1996;4:180.

26. Kalvach et al. Geriatrie a gerontologie. Praha: Grada Publishing, 2004.

27. Kang HG, Dingwell JB. A direct Comparison of Local Dynamics Stability During Upright Standing and walking. Experimental Brain Research 2006; 172(2):35-48.

28. Kapteyn TS, Bles W, Njiokiktjien ChJ, Kodde L, Massen CH, Mol JMF. Standardization in Platform Stabilometry being a Part of Posturography. Agressologie 1983;24(7): 321-326

29. Kapteyn TS. Afterthought about the physics and Mechanics of the postural sway. Agressologie, 1973;14C:27-35

30. Kelley TL. Interpretation of educational measurements. Yonkers (NY): World books, 1927.

31. Kidder SMS, Abuzzahab FMS, Dow A Ortiz TBS, Harris G, Johnson JMD Repeatability of postural data in normal foot and ankle motion. Gait and Posture $1996 ; 4: 180$

32. Koozenkanani M, Duerk H. Determination of body segment parameters and their effect in the calculation of the position of COP during postural sway. IEEE Transactions on Biomedical Engineering 1985:32:67-69.

33. Lafond $\mathrm{H}$, Corriveau $\mathrm{H}$, Hebert R, Prince F. Intrassession reliability of COP measures of postural steadiness in healthy elderly people. Arch. Of Phys. Med. Reh 2004;85:896-901.

34. Laughton CA, Slavin M, Katdare K, Nolan L, Bean JF, Kerrigan DC, Phillips E, Lipsitz LA, Collins JJ. Aging, muscle activity and balance control: physiologic changes associated with balance impairment. Gait and Posture 2004;18:101-108.

35. LeClair K, Riach C. Postural stability measurement: test duration and outcome parameters. In: Woolacott M, Horak F, editors Posture and Gait: control mechanisms vol. 1. Oregon University of Oregon Books 1992;400-3.

36. Lenhoff MW, Santner TJ, Peterson MGE, Daly L. Bootstrap simultaneous prediction and confidence intervals for gait data. Gait and Posture 1996;4:172.

37. Letz R, Gerr F. Standing steadiness measurements: empirical selection of testing protocols and outcome measures. Neurotoxicol teratol 1995;17:611-616.

38. Little C, Patla EA, Winter DA, Riedtyk S, Judge S. Unexpected trunk perturbations reveal considerable complexity in postural control. Proceedeings $13^{\text {th }}$ Symposium Int. for Postural and Gait Research, Paris, June 22-26, 1997.

39. MacKinnon CD, Winter DA. Control of whole body balance and posture in the frontal plane during walking. Journal of Biomechanics 1993;26:633-644.

40. Megrot F, Bardy BG, Dietrich G. Dimensionality and the dynamics of human unstable equilibrium. Journal of motor behavior 2002;34:323-328.

41. Menz BH, Lord SR, Fitzpatrick RC. Age-related differences in walking stability. Age and Ageing 2003; 32(2): 137-142.

42. Morris JN, Hardman AE. Walking to health. Sports Med 1997;23:306-32.

43. Overstall PW, Johnson AL, Exton-Smith AN. Instability and falls in the elderly. Age and Aging 1978;7:92-96.
44. Powel GN, Dzendolet E. Power spectral density analyses of lateral human standing sway. Journal of motor behavior 1984;16:424-441.

45. Prieto TE, Myklebust JB, Hoffmann RG, Lowett EG, Myklebust BM. Measures of postural steadiness: defferences between healthy young and elderly adults. IEE Transactions on Biomedical Engineering 1996;43:956-966.

46. Prince F, Winter DA, Archer SE. Assesment of postural control during quiet stance with different foot configurations. Gait and Posture 1996;4:110.

47. Raz RC. Learning disabilities and postural control. Freund Publishing House LTD, London, 1996.

48. Riley PO. Modelling of the biomechanics of posture and balance. Journal of Biomechanics 1990;23:503-506.

49. Romberg MH. Manual of nervous diseases of man, London: Sydeham Society, 1953

50. Samson M, Crowe A. Intra-subject inconsistecies in quantitative assessments of body sway. Gait and Posture 1996;4:252-257.

51. Skinner HB, Barak RL, Cook SD. Age-related declines in proprioception. Clinical Orthopaedics 1988;184:208-211.

52. Spaepen AJ, VranKen M, Williams EJ. Comparison of the movements of the COG and COP in stabilometric studies. Agressologie 1977;18:109-113.

53. Spirduso WW. Physical dimensions of aging. Human Kinetics Publisher, 1995.

54. Tinetti ME, Richman D, Powel L. Falls efficacy as a measure of fear of falling Journal of Gerontology, Psychological science 1990;54:P239-243.

55. Tobis JS, Nayak L, Hochler FK. Visual perception verticality and horizontality among fallers. Arch. Of Phys Med Reh 1981;62:619-622.

56. Topinková E. Geriatrie pro praxi, Praha: Galén, 2005.

57. Tošnerová $\mathrm{V}$, Hvězdová J, Miláček Z. Výsledky vyšetření na stabilometrické plošině na rehabilitační klinice v Hradci Králové. Rehabilitace a fyzikální lékařstvi 2004;11:118-121

58. Véle F. Kineziologie pro klinickou praxi. Praha: Grada, 1997.

59. Weiner EA, Stewart BJ. Assessing individuals. Boston (MA): Little Brown, 1984

60. Winter D, Patla AE, Prince F, Ishac M. Stiffness Control of Balance in Quiet Standing. Journal of Neurophysiology 1998;80:1211-1221.

61. Winter DA, Prince F, Frank JS, Powel C, Zabjek KF. Unified Theory Regarding the $\mathrm{A} / \mathrm{P}$ and $\mathrm{M} / \mathrm{L}$ Balance in Quiet Stance, Journal of Neurophysiology 1996;75:2334-2343.

62. Winter DA, Prince F, Patla A. Interpretation of COM and COP balance Control during quiet standing Gait and Posture 1996;4:174

63. Winter DA. ABC of balance during standing and walking. Waterloo: Waterloo biomechanics, 1995 .

64. Winter DA. Human balance and posture control during standing and walking Gait and Posture 1995;3:193-214.

65. Wolley S, McCarter J, Randolf B. An assesment of foam support surfaces used in static stabilometry. Gait and Posture 1996;4:110.

66. Woollacoott MH, Shumway-Cook A, Nashner L. Aging and posture control changes in sensory organisation and muscular coordination. International journal of aging and human development 1986;23:97-114

67. Yang CT. A Study on the Robust Control of an Inverted Pendulum Using Discrete Sliding-Mode Control. Springer Berlin: Heidelberg, 2006.

68. Yunacheng J, Winter DA, Ishac MG, Gilchrist L. Trajectory of the body COP and COG during initiation and termination of gait. Gait and Posture 1993; $1: 9-22$

Submitted January 2008. Accepted September 2008.

\section{Corresponding author:}

Jitka Jančová, MS, Charles University in Prague, Faculty of Physical Education and Sport, Department of Sport Medicine and Adapted Physical Education, José Martiho 31, Praha 6, Czech Republic, e-mail: jitka.vseteckova@post.cz 